\title{
Editorial: Lasers in Accelerator Science and Secondary Emission Light Source Technology
}

\author{
Sergio Carbajo ${ }^{1 *}$, Arya Fallahi ${ }^{2,3}$, Jerome Faure ${ }^{4}$ and Liang Jie Wong ${ }^{5,6}$ \\ ${ }^{1}$ SLAC National Accelerator Laboratory, Stanford University, Menlo Park, CA, United States, ${ }^{2}$ IT'IS Foundation, Zurich, \\ Switzerland, ${ }^{3}$ Swiss Federal Institute of Technology, ETH Zurich, Zurich, Switzerland, ${ }^{4}$ Laboratoire d'Optique Appliquée and \\ École Polytechnique, Palaiseau, France, ${ }^{5}$ School of Electrical and Electronic Engineering, Nanyang Technological University, \\ Singapore, Singapore, ${ }^{6}$ Singapore Institute of Manufacturing Technology, Agency for Science, Technology and Research \\ (A*STAR), Singapore, Singapore
}

Keywords: laser accelerated particles, X-ray emission, free electron laser (FEL), ultrafast optics, secondary emission and photoemission, non-linear optics

\section{Editorial on the Research Topic}

\section{Lasers in Accelerator Science and Secondary Emission Light Source Technology}

Unifying laser and accelerator physics holds great promise for the development of future particle accelerators, light sources, and other scientific instruments due to increasingly synergistic advances at the cross section between these two fields. Their combined action has recently ushered in advanced accelerator facilities around the world that have enabled unique scientific and technological breakthroughs: from advanced electron and ion sources for high-energy physics to the ultrabright $\mathrm{x}$-ray pulses to study ultrafast phenomena at elemental spatio-temporal scales [1-4]. The progress of ultra-intense femtosecond lasers, now attaining multi PetaWatt peak power, has recently enabled the demonstration of $\mathrm{GeV}$ electron beams in centimeter scale plasma accelerating section [5, 6], with the recent world record reaching $8 \mathrm{GeV}$ in $20 \mathrm{~cm}$ [7]. As for ultrafast x-ray science, in their relatively short time since their advent, x-ray free electron lasers [8, 9] (FEL) have demonstrated the capacity to answer grand fundamental questions in a diverse set of areas in physics, chemistry, and biology, such as revealing vibration coherence in molecules [10], molecular bond formation, charge migration, and dissociation dynamics [11, 12], or ultrafast isomerization in biomolecules [13, 14], among many others. Further advances in facilities-such as augmented brightness, attosecond duration, or seeded emission - are poised to creating new scientific frontiers in atomic-scale correlated systems and ultrahigh resolution inner shell spectroscopies.

Future laser-based or laser-enabled accelerator and light source technologies could tap into an incredibly broad range of methodologies, including but not limited to well-underway approaches like laser wakefield acceleration [15-18] and seeded [9] and echo-enabled harmonic generation in FELs $[19,20]$ as well as more exploratory compact accelerator concepts such as free-space [21-23], terahertz [24-26], and on-chip [27-29] accelerators, among many others. Rather than a competition for the best possible future technology, these approaches answer to vastly differing scientific and technological needs, from large-scale colliders for exploration of fundamental physics $[16,30]$ to compact systems for radiation therapy [27], to name a few. In all cases, lasers constitute essential accelerator components, and much of the progress in both fields strides alongside of one another. This is because laser fields are ideal agents to generate and tailor the 6-D phase-space distribution of charged particle beams with extremely high spatio-temporal precision. For example, lasers can readily generate charged particles via photoemission or photoinization, and be used in ultrafast manipulation and beam diagnostics [31,32]. Lasers are also integral to accelerator-based light sources XFELs and their applications in ultrafast sciences [33, 34]. 
Most accelerators share common needs in driving laser requirements: high peak and average power, and high wallplug efficiency. Increasing the peak power enables ultrahigh-field regimes to understand fundamental matter-light interactions for particle acceleration, while average power and wall-plug efficiency are essential for bright light and particle sources for practical accelerator applications outside of basic sciences, such as systems for medicine, industrial applications, and defense and security. However, outside of these general requirements, the myriads of potential laser-accelerator configurations highlight the difficulty in encapsulating all the research directions capable of tackling critical technical challenges for future accelerator and light source technology.

Through this Research Topic, our intent is to showcase advances in various parallel topical areas that collectively fill the breach between laser and accelerator physics. This issue exposes some present and future opportunities in laser research and development that serves accelerator technology. Our hope is that the contributed works will help identify cooperative international research initiatives to expedite progress in lasers for accelerator science and secondary emission light source technology.

One of the sub-topical areas of this topic covers compact laserbased acceleration. The paper by Wilhelm and Durfee follows current trends of advanced laser manipulation for optimizing the interaction of a laser pulse with electrons in vacuum. This work utilizes spatio-temporal couplings in order to facilitate the acceleration of electrons in vacuum. The authors show that by introducing a pulse front tilt in the laser pulse, electron trapping, and acceleration is more efficient compared to the use of traditional Gaussian pulses. The scheme is studied in the context of small lab-scale table-top laser systems and aims at producing quality electron beams at the $\mathrm{MeV}$ level. In addition to pulse front, the temporal chirp of laser pulses also plays an important role in particle acceleration and developing accurate and simple models for the propagation of such pulses is of an utmost importance. Salamin and Carbajo present a simple model

\section{REFERENCES}

1. Bostedt C, Boutet S, Fritz DM, Huang Z, Lee HJ, Lemke HT, et al. Linac coherent light source: the first five years. Rev Mod Phys. (2016) 88:15007. doi: 10.1103/RevModPhys.88.015007

2. Pile D. First light from SACLA. Nat Photonics. (2011) 5:456. doi: $10.1038 /$ nphoton.2011.178

3. Hogan MJ, Raubenheimer TO, Seryi A, Muggli P, Katsouleas T, Huang C, et al. Plasma wakefield acceleration experiments at FACET. New J Phys. (2010) 12:55030. doi: 10.1088/1367-2630/12/5/055030

4. McNeil B. First light from hard X-ray laser. Nat Photonics. (2009) 3:375. doi: $10.1038 /$ nphoton.2009.110

5. Kim HT, Pae KH, Cha HJ, Kim IJ, Yu TJ, Sung JH, et al. Enhancement of electron energy to the multi-GeV regime by a dual-stage laser-wakefield accelerator pumped by petawatt laser pulses. Phys Rev Lett. (2013) 111:165002. doi: 10.1103/PhysRevLett.111.165002

6. Wang X, Zgadzaj R, Fazel N, Li Z, Yi SA, Zhang X, et al. Quasi-monoenergetic laser-plasma acceleration of electrons to $2 \mathrm{GeV}$. Nat Commun. (2013) 4:1988. doi: 10.1038/ncomms 2988

7. Gonsalves AJ, Nakamura K, Daniels J, Benedetti C, Pieronek C, de Raadt TCH, et al. Petawatt laser guiding and electron beam acceleration to $8 \mathrm{GeV}$ in a for the fields of a chirped laser pulse and used the model to investigate electron acceleration with chirped pulses.

As laser wakefield acceleration is reaching technological maturity, Alejo et al. present a review of the potential applications in the generation of secondary sources that these new laser driven electron beams now permit. X-ray beams at the $\mathrm{MeV}$ level have been generated by inverse Compton scattering, leading to some of the highest brightness for a photon source in this energy range. The authors also discuss the potential of laser wakefield accelerators for positron generation via the BetheHeitler mechanism, showing promising prospects in terms of charge and beam quality.

As for secondary emission sources, Hemsing presents a theory for echo-enabled harmonic generation (EEHG) that considers finite laser pulse durations, revealing conditions under which the bandwidth of the harmonic bunching peak is minimized in the presence of arbitrary phase distortions. In a collaboration between scientists at Helmholtz-Zentrum DresdenRossendorf and Technical University of Dresden, Steiniger et al. present a novel concept based on Thomson scattering off a laser with tilted pulse front. It is theoretically shown that the new phenomenon, named as Traveling-Wave Thomson Scattering (TWTS) enables enhancement of the incoherent radiation compared to a conventional Thomson scattering. The work presents a conceptual design of optical setups and the overall requirements based on the state-of-the-art laser technology. Finally, the work by Tibai et al. shows that isolated waveform-controlled extreme ultraviolet attosecond pulses can be produced under realistic experimental conditions, using a method based on coherent undulator radiation of relativistic ultrathin electron layers.

\section{AUTHOR CONTRIBUTIONS}

All authors listed have made a substantial, direct and intellectual contribution to the work, and approved it for publication.

laser-heated capillary discharge waveguide. Phys Rev Lett. (2019) 122:84801 doi: 10.1103/PhysRevLett.122.084801

8. Ishikawa T, Aoyagi H, Asaka T, Asano Y, Azumi N, Bizen T, et al. A compact $\mathrm{X}$-ray free-electron laser emitting in the sub-ångström region. Nat Photonics. (2012) 6:540-4. doi: 10.1038/nphoton.2012.141

9. Amann J, Berg W, Blank VD, Decker F-J, Ding Y, Emma P, et al. Demonstration of self-seeding in a hard-X-ray free-electron laser. Nat. Photonics. (2012) 6:693-8. doi: 10.1038/nphoton.2012.180

10. Stankus B, Yong H, Zotev N, Ruddock JM, Bellshaw D, Lane TJ, et al. Ultrafast X-ray scattering reveals vibrational coherence following Rydberg excitation. Nat Chem. (2019) 11:716-21. doi: 10.1038/s41557-019-0 291-0

11. Ruddock JM, Zotev N, Stankus B, Yong H, Bellshaw D, Boutet S, et al. Simplicity beneath complexity: counting molecular electrons reveals transients and kinetics of photodissociation reactions. Angew Chem. (2019) 58:6371-5. doi: 10.1002/ange.201902228

12. Kern J, Chatterjee R, Young ID, Fuller FD, Lassalle L, Ibrahim M, et al. Structures of the intermediates of Kok's photosynthetic water oxidation clock. Nature. (2018) 563:421-5. doi: 10.1038/s41586-018-0681-2

13. Coquelle N, Sliwa M, Woodhouse J, Schirò G, Adam V, Aquila A, et al. Chromophore twisting in the excited state of a photoswitchable fluorescent 
protein captured by time-resolved serial femtosecond crystallography. Nat Chem. (2018) 10:31-7. doi: 10.1038/nchem.2853

14. Nogly P, Weinert T, James D, Carbajo S, Ozerov D, Furrer A, et al. Retinal isomerization in bacteriorhodopsin captured by a femtosecond $\mathrm{x}$-ray laser. Science. (2018) 361:eaat0094. doi: 10.1126/science.aat0094

15. Geddes CG, Toth CS, Van Tilborg J, Esarey E, Schroeder CB, Bruhwiler D, et al. High-quality electron beams from a laser wakefield accelerator using plasma-channel guiding. Nature. (2004) 431:538-41. doi: $10.1038 /$ nature 02900

16. Faure J, Glinec Y, Pukhov A, Kiselev S, Gordienko S, Lefebvre E, et al. A laserplasma accelerator producing monoenergetic electron beams. Nature. (2004) 431:541-4. doi: 10.1038/nature02963

17. Esarey E, Schroeder CB, Leemans WP. Physics of laser-driven plasma-based electron accelerators. Rev Mod Phys. (2009) 81:1229. doi: 10.1103/RevModPhys.81.1229

18. Mangles SP, Murphy CD, Najmudin Z, Thomas AG, Collier JL, Dangor AE, et al. Monoenergetic beams of relativistic electrons from intense laser-plasma interactions. Nature. (2004) 431:535. doi: 10.1038/nature02939

19. Hemsing E, Dunning M, Garcia B, Hast C, Raubenheimer T, Stupakov G, et al. Echo-enabled harmonics up to the 75th order from precisely tailored electron beams. Nat Photonics. (2016) 10:512-5. doi: 10.1038/nphoton.2016.101

20. Ribič PR, Roussel E, Penn G, De Ninno G, Giannessi L, Penco G, et al. Echo-enabled harmonic generation studies for the FERMI free-electron laser. Photonics. (2017) 4:19. doi: 10.3390/photonics4010019

21. Wong LJ, Kärtner FX. Direct acceleration of an electron in infinite vacuum by a pulsed radially-polarized laser beam. Opt. Express. (2010) 18:25035-51. doi: 10.1364/OE.18.025035

22. Carbajo S, Nanni EA, Wong LJ, Moriena G, Keathley PD, Laurent G, et al. Direct longitudinal laser acceleration of electrons in free space. Phys Rev Accel Beams. (2016) 19:21303. doi: 10.1103/PhysRevAccelBeams.19.021303

23. Wong LJ, Hong KH, Carbajo S, Fallahi A, Piot P, Soljačić M, et al. Laserinduced linear-field particle acceleration in free space. Sci. Rep. (2017) 7:11159. doi: 10.1038/s41598-017-11547-9

24. Kärtner FX, Ahr F, Calendron AL, Çankaya H, Carbajo S, Chang G, et al. AXSIS: exploring the frontiers in attosecond X-ray science, imaging and spectroscopy. Nucl Instrum Methods Phys Res Sect A Accel Spectrom Detect Assoc Equip. (2016) 829:24-9. doi: 10.1016/j.nima.2016.02.080

25. Nanni EA, Huang WR, Hong KH, Ravi K, Fallahi A, Moriena G, et al. Terahertz-driven linear electron acceleration. Nat Commun. (2015) 6:8486. doi: 10.1038 /ncomms 9486
26. Zhang D, Fallahi A, Hemmer M, Wu X, Fakhari M, Hua Y, et al. Segmented terahertz electron accelerator and manipulator (STEAM). Nat Photonics. (2018) 12:336-42. doi: 10.1038/s41566-018-0138-z

27. England RJ, Noble RJ, Bane K, Dowell DH, Ng C-K, Spencer JE, et al. Dielectric laser accelerators. Rev Mod Phys. (2014) 86:1337-89. doi: 10.1103/RevModPhys.86.1337

28. Peralta EA, Soong K, England RJ, Colby ER, Wu Z, Montazeri B, et al. Demonstration of electron acceleration in a laser-driven dielectric microstructure. Nature. (2013) 503:91. doi: 10.1038/nature12664

29. McNeur J, Kozák M, Schönenberger N, Leedle KJ, Deng H, Ceballos A, et al. Elements of a dielectric laser accelerator. Optica. (2018) 5:687-90. doi: 10.1364/OPTICA.5.000687

30. Leemans WP, Nagler B, Gonsalves AJ, Tóth C, Nakamura K, Geddes CGR, et al. GeV electron beams from a centimetre-scale accelerator. Nat Phys. (2006) 2:696-9. doi: 10.1038/nphys418

31. Kirchner FO, Gliserin A, Krausz F, Baum P. Laser streaking of free electrons at $25 \mathrm{keV}$. Nat Photonics. (2013) 8:52. doi: 10.1038/nphoton.2013.315

32. Eckle P, Smolarski M, Schlup P, Biegert J, Staudte A, Schöffler M, et al. Attosecond angular streaking. Nat Phys. (2008) 4:565-70. doi: $10.1038 /$ nphys 982

33. Liebster N, Tang J, Ratner D, Liu W, Vetter S, Huang Z, et al. Laguerre-Gaussian and beamlet array as second generation laser heater profiles. Phys Rev Accel Beams. (2018) 21:90701. doi: 10.1103/PhysRevAccelBeams.21.090701

34. Sierra RG, Batyuk A, Sun Z, Aquila A, Hunter MS, Lane TJ, et al. The macromolecular femtosecond crystallography instrument at the linac coherent light source. J. Synchrotron Radiat. (2019) 26:346-57. doi: $10.1107 /$ S1600577519001577

Conflict of Interest: The authors declare that the research was conducted in the absence of any commercial or financial relationships that could be construed as a potential conflict of interest.

Copyright (c) 2019 Carbajo, Fallahi, Faure and Wong. This is an open-access article distributed under the terms of the Creative Commons Attribution License (CC BY). The use, distribution or reproduction in other forums is permitted, provided the original author(s) and the copyright owner(s) are credited and that the original publication in this journal is cited, in accordance with accepted academic practice. No use, distribution or reproduction is permitted which does not comply with these terms. 